\title{
A multifunction filter configuration based on CFA
}

\author{
Pinghua Zhang ${ }^{1, \text { a }}$, Zhihui Deng ${ }^{2, b}$ \\ ${ }^{1}$ Hunan College of Information, Changsha, Hunan, China \\ ${ }^{2}$ Hunan College of Information, Changsha, Hunan, China \\ apinghua1201@126.com, ’dengzhihui@mail.hniu.cn
}

Keywords: Current feedback amplifier; Filter; Multifunction configuration

Abstract. A new multifunction filter configuration using two CFAs and six impedances is presented in this paper. The proposed circuit can realize the different function using the different impedances. The natural frequency $\omega 0$ and quality factor $\mathrm{Q}$ can be orthogonal controlled, and the quality factor $\mathrm{Q}$ can also be independently controlled by the impedance. Both the active and passive sensitivities are no more than unity. At the end, the theoretical analysis is verified using PIPICE simulations of the filter designs.

\section{Introduction}

In analog signal processing, the current mode circuits have drawn more and more attentions. Many current mode circuits have been put forward recently, such as static current mirror, cross wire circuit, current transmitter, current feedback operational amplifier (transimpedance operational amplifier), etc. Strictly speaking, current transmitter and current feedback amplifier should be the current/voltage hybrid circuits ${ }^{[1]}$. However, the current mode is the main, and plays a main role in the current performance. Therefore, put them in the current mode circuits.

Current Feedback Operational Amplifier (CFA for short), also called transimpedance Operational Amplifier, it is a new type of ultra high speed amplifier which developed very quickly in the early $1990 \mathrm{~s}^{[2]}$. When the negative Feedback closed loop works, the input feeds in low resistance, the feedback of the Reversed-Phase input is current, it is a kind of transimpedance amplifiers with input voltage buffer. Compared with the traditional voltage mode operation amplifier also named Voltage Feedback Operational Amplifier (VFA for short), it has completely different topology and working principle, and it is the latest achievement of integrated operational amplifier ${ }^{[3]}$. In this paper, the structure of a multifunction filter is composed of two CFA and six impedance elements. The function of the filter can be realized by changing the impedance elements without changing the structure. The natural frequency $\omega_{0}$ and quality factor $Q$ can be orthogonal controlled, and the quality factor $Q$ can also be independently controlled by the impedance. Both the active and passive sensitivities are no more than unity. The results obtained from the PSPICE simulations are consistent with the theoretical analysis.

\section{Port Properties of Current Feedback Amplifier}

As a four terminal network, CFA's ideal port properties is ${ }^{[4]}$

$$
\left(\begin{array}{l}
V_{x} \\
I_{y} \\
I_{z}
\end{array}\right)=\left(\begin{array}{ccc}
1 & 0 & 0 \\
0 & 0 & 0 \\
0 & 0 & \pm 1
\end{array}\right)\left(\begin{array}{l}
V_{y} \\
V_{z} \\
I_{x}
\end{array}\right) \text {, and } V_{o}=V_{z}
$$

Its representative symbol is shown in Fig. 1, where " $\mathrm{x}$ " is Reversed-Phase input (current input), "Y" is in-phase input (voltage input). "+" for CFA+, "-" for CFA-. 


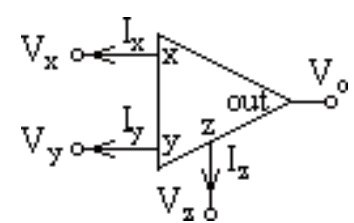

Fig.1. Symbol of CFA

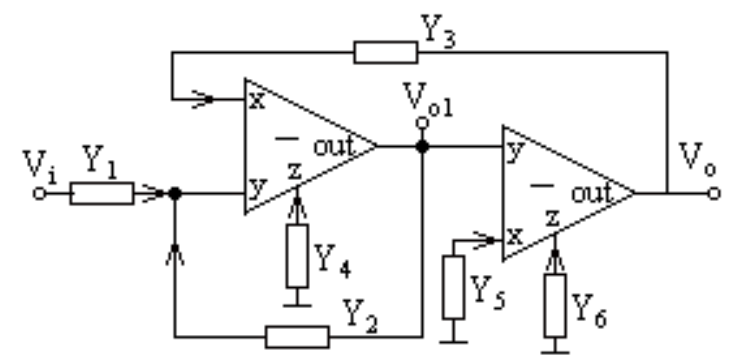

Fig.2. Multifunction Filter Configuration

\section{Circuit Description}

The structure of the multifunction filter with two CFA- and six impedance elements is shown in Fig. 2. According to the circuit of Fig. 2 and the port properties of CFA, it is available ${ }^{[5]}$.

$$
V_{o}=\frac{Y_{1} Y_{3} Y_{5}}{D} V_{1} \quad V_{o 1}=-\frac{Y_{1} Y_{3} Y_{6}}{D} V_{1}
$$

Where

$$
D=Y_{1} Y_{3} Y_{5}+Y_{2} Y_{3} Y_{5}+Y_{2} Y_{3} Y_{6}+Y_{1} Y_{4} Y_{6}+Y_{2} Y_{4} Y_{6}
$$

Considering the non ideal characteristic of CFA:

$$
I_{z}=\alpha I_{x}, V_{x}=\beta V_{y}, V_{o}=\gamma V_{z}
$$

Where $\alpha=1-\varepsilon_{1}\left(\varepsilon_{1}<<1\right)$ is current transmission error of CFA, $\beta=1-\varepsilon_{2}\left(\varepsilon_{2}<<1\right)$ is input voltage transmission error, $\gamma=1-\varepsilon_{3}\left(\varepsilon_{3}<<1\right)$ is output voltage transmission error. Transmission function of the filter can be rewritten as ${ }^{[6]}$ :

$$
V_{o}=\frac{\alpha_{1} \alpha_{2} \beta_{1} \beta_{2} \gamma_{1} \gamma_{2} Y_{1} Y_{3} Y_{5}}{T} V_{1}, V_{o l}=-\frac{\alpha_{1} \beta_{1} \gamma_{1} \gamma_{2} Y_{1} Y_{3} Y_{6}}{T} V_{1}
$$

Where

$$
\mathrm{T}=\alpha_{1} \alpha_{2} \beta_{2} \gamma_{1} \gamma_{2} \mathrm{Y}_{1} \mathrm{Y}_{3} \mathrm{Y}_{5}+\alpha_{1} \alpha_{2} \beta_{1} \beta_{2} \gamma_{1} \gamma_{2} \mathrm{Y}_{2} \mathrm{Y}_{3} \mathrm{Y}_{5}+\alpha_{1} \beta_{1} \gamma_{1} \gamma_{2} \mathrm{Y}_{2} \mathrm{Y}_{3} \mathrm{Y}_{6}+\mathrm{Y}_{1} \mathrm{Y}_{4} \mathrm{Y}_{6}+\beta_{1} \mathrm{Y}_{2} \mathrm{Y}_{4} \mathrm{Y}_{6}
$$

When the six impedance elements are different, the functions are also different:

(1) When $Y_{4}=S C_{4}, Y_{6}=S C_{6}$, the rest of the components are resistance, the circuit can achieve two order low-pass and band-pass filter, the transmission function of the circuit is written as:

$$
V_{o}=\frac{G_{1} G_{3} G_{5}}{D_{1}} V_{1}, V_{o l}=-\frac{S G_{1} G_{3} C_{6}}{D_{1}} V_{1}
$$

Where $D$ can be rewritten as

$$
D_{1}=S^{2} C_{4} C_{6}\left(G_{1}+G_{2}\right)+S C_{6} G_{2} G_{3}+\left(G_{1}+G_{2}\right) G_{3} G_{5}
$$

The natural frequency $\omega_{0}$ and quality factor $Q$ :

$$
\omega_{o}=\frac{1}{\sqrt{C_{4} C_{6} R_{3} R_{5}}}, Q=\left(1+\frac{R_{2}}{R_{1}}\right) \sqrt{\frac{C_{4} R_{3}}{C_{6} R_{5}}}
$$

Considering the non ideal characteristic of CFA, the natural frequency $\omega_{0}$ and quality factor $Q$ : 


$$
\omega_{o}=\sqrt{\frac{\alpha_{1} \alpha_{2} \beta_{2} \gamma_{1} \gamma_{2}}{C_{4} C_{6} R_{3} R_{5}}}, Q=\left(1+\frac{R_{2}}{\beta_{1} R_{1}}\right) \sqrt{\frac{\alpha_{2} \beta_{2} C_{4} R_{3}}{\alpha_{1} \gamma_{1} \gamma_{2} C_{6} R_{5}}}
$$

The sensitivities of passive and active components caused by $\omega_{o}, Q$ are respectively:

$$
\begin{aligned}
& S_{C_{4}, C_{6}, R_{3}, R_{5}}^{\omega_{o}}=-\frac{1}{2}, S_{C_{4}, R_{3}}^{Q}=\frac{1}{2}=-S_{C_{6}, R_{5}}^{Q}, S_{R_{2}}^{Q}=1=-S_{R_{l}}^{Q}, S_{\alpha_{1}, \alpha_{2}, \beta_{2}, \gamma_{1}, \gamma_{2}}^{\omega_{o}}=\frac{1}{2}, \\
& S_{\beta_{1}}^{Q}=-1, S_{\alpha_{2}, \beta_{2}}^{Q}=\frac{1}{2}=-S_{\alpha_{1}, \gamma_{1}, \gamma_{2}}^{Q}
\end{aligned}
$$

(2) When $Y_{3}=S C_{3}, Y_{5}=S C_{5}$, the rest of the components are resistance, the circuit can achieve two order high-pass and band-pass filter, the transmission function of the circuit is written as:

$$
V_{o}=\frac{S^{2} G_{1} C_{3} C_{5}}{D_{2}} V_{1}, V_{o l}=-\frac{S G_{1} C_{3} G_{6}}{D_{2}} V_{1}
$$

Where $D$ can be rewritten as

$$
D_{2}=S^{2} C_{3} C_{5}\left(G_{1}+G_{2}\right)+S C_{3} G_{2} G_{6}+\left(G_{1}+G_{2}\right) G_{4} G_{6}
$$

The natural frequency $\omega_{0}$ and quality factor $Q$ :

$$
\omega_{0}=\frac{1}{\sqrt{C_{3} C_{5} R_{4} R_{6}}}, Q=\left(1+\frac{R_{2}}{R_{1}}\right) \sqrt{\frac{C_{5} R_{6}}{C_{3} R_{4}}}
$$

Considering the non ideal characteristic of CFA, the natural frequency $\omega_{0}$ and quality factor $Q$ :

$$
\omega_{o}=\frac{1}{\sqrt{\alpha_{1} \alpha_{2} \beta_{2} \gamma_{1} \gamma_{2} C_{3} C_{5} R_{4} R_{6}}}, Q=\left(1+\frac{R_{2}}{\beta_{1} R_{1}}\right) \sqrt{\frac{\alpha_{2} \beta_{2} C_{5} R_{6}}{\alpha_{1} \gamma_{1} \gamma_{2} C_{3} R_{4}}}
$$

The sensitivities of passive and active components caused by $\omega_{o}, Q$ are respectively:

$$
\begin{aligned}
& S_{C_{3}, C_{5}, R_{4}, R_{6}}^{\omega_{o}}=-\frac{1}{2}, S_{C_{5}, R_{6}}^{Q}=\frac{1}{2}=-S_{C_{3}, R_{4}}^{Q}, S_{R_{2}}^{Q}=1=-S_{R_{1}}^{Q}, S_{\alpha_{1}, \alpha_{2}, \beta_{2}, \gamma_{1}, \gamma_{2}}^{\omega_{o}}=-\frac{1}{2}, \\
& S_{\beta_{l}}^{Q}=-1, S_{\alpha_{2}, \beta_{2}}^{Q}=\frac{1}{2}=-S_{\alpha_{1}, \gamma_{1}, \gamma_{2}}^{Q}
\end{aligned}
$$

It is known from Eq. (9)(10)and Eq.(14)(15), for the two cases, the natural frequency $\omega_{0}$ and quality factor $Q$ of the filter can be orthogonal controlled by impedance elements $Y_{3}, Y_{4}, Y_{5}$ and $Y_{6}$ independently, and quality factor $Q$ can also is controlled by resistors $R_{1}$ and $R_{2}$ separately. Moreover, both the active and passive sensitivities are no more than unity.

(3) When $Y_{2}=S C_{2}, Y_{3}=S C_{3}$, the rest of the components are resistance, both the outputs of the circuit can achieve two order band-pass filter. While the previous two cases has been able to achieve band-pass filtering, so the third case need not be considered.

The circuit structure is designed to achieve the function of the two order filter, and this structure can also achieve the three order filter:

(4)When $Y_{1}=S C_{1}, Y_{3}=S C_{3}, Y_{5}=S C_{5}$, the rest of the components are resistance, the circuit can achieve three order high-pass and band-pass filter, the transmission function of the circuit is written as:

$$
V_{o}=\frac{S^{3} C_{1} C_{3} C_{5}}{D_{4}} V_{1}, V_{o l}=-\frac{S^{2} C_{1} C_{3} G_{6}}{D_{4}} V_{1}
$$


Where $D$ can be rewritten as

$$
D_{4}=S^{3} C_{1} C_{3} C_{5}+S^{2} C_{3} C_{5} G_{2}+S G_{5}\left(C_{3} G_{2}+C_{1} G_{4}\right)+G_{2} G_{4} G_{6}
$$

(5)When $Y_{2}=S C_{2}, Y_{3}=S C_{3}, Y_{5}=S C_{5}$, the rest of the components are resistance, the circuit can achieve three order high-pass and band-pass filter, the transmission function of the circuit is written as:

$$
V_{o}=\frac{S^{2} G_{1} C_{3} C_{5}}{D_{5}} V_{l}, V_{o l}=-\frac{S G_{1} C_{3} G_{6}}{D_{5}} V_{l}
$$

Where $D$ can be rewritten as

$$
D_{5}=S^{3} C_{2} C_{3} C_{5}+S^{2} C_{3}\left(C_{5} G_{I}+C_{2} G_{6}\right)+S C_{2} G_{4} G_{6}+G_{I} G_{4} G_{6}
$$

(6) When $Y_{2}=S C_{2}, Y_{4}=S C_{4}, Y_{6}=S C_{6}$, the rest of the components are resistance, the circuit can achieve three order low-pass and band-pass filter, the transmission function of the circuit is written as:

$$
V_{o}=\frac{S^{3} G_{1} G_{3} G_{5}}{D_{6}} V_{1}, \quad V_{o l}=-\frac{S^{2} G_{1} G_{3} C_{6}}{D_{6}} V_{1}
$$

Where $D$ can be rewritten as

$$
D_{6}=S^{3} C_{2} C_{4} C_{6}+S^{2} C_{6}\left(C_{4} G_{1}+C_{2} G_{3}\right)+S C_{2} G_{3} G_{5}+G_{I} G_{3} G_{5}
$$

\section{Computer Simulation}

The two order circuit is simulated using PSPICE. In case 1 , when $R_{i}=10 \mathrm{k} \Omega$, $(i=1,2,3,5), C_{4}=C_{6}=1.59 \mathrm{nF}$. In case 2 , when $R_{i}=10 \mathrm{k} \Omega,(i=1,2,4,6), C_{3}=C_{5}=1.59 \mathrm{nF}$, The response curves can be obtained respectively as shown in Fig. 3 and Fig.4.

In case 1 , when $R_{i}=10 \mathrm{k} \Omega,(i=1,2,3,5)$,change the value of $C_{4}$ and $C_{6}$, observing the change of the natural frequency $\omega_{0}$ and quality factor $Q$. From Fig. 5 and Fig. 6, which can be seen that the natural frequency $\omega_{0}$ and quality factor $Q$ can be orthogonal controlled by capacitance $C_{4}$ and $C_{6}$.

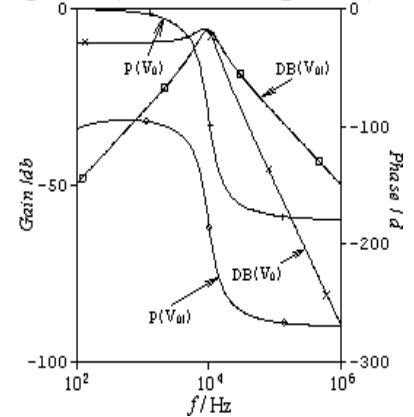

Fig.3. Response curves In case 1

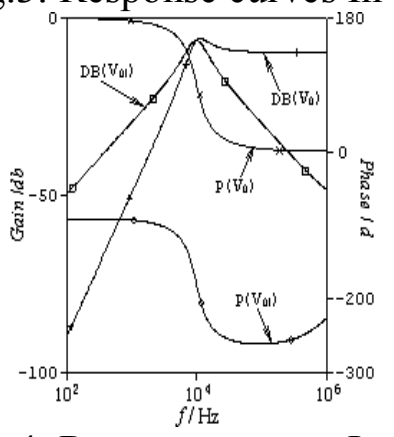

Fig.4. Response curves In case 2

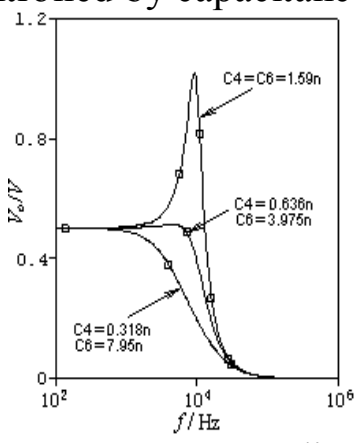

Fig.5.Low-pass amplitude-frequency curves

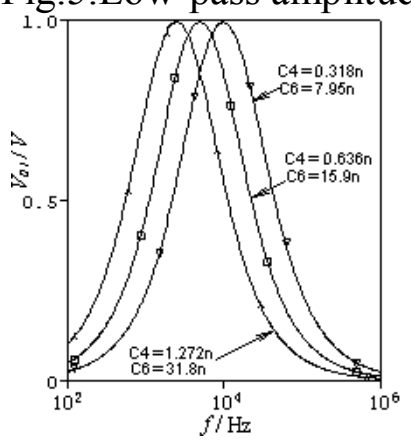

Fig.6.Band-pass amplitude-frequency curves 


\section{Conclusions}

In this paper, a multifunction filter is designed with two CFA- and six impedance elements. Without changing the circuit structure, the different filter function can be realized by changing the impedance elements. The natural frequency $\omega 0$ and quality factor $Q$ can be orthogonal controlled, and the quality factor $Q$ can also be independently controlled by the impedance. Both the active and passive sensitivities are no more than unity. Although the quality factor Q can be adjusted by the impedance element, but at the same time, it will affect the amplitude of the output signal. At the end, the circuit is simulated by PSPICE. The simulation results are in agreement with the theoretical analysis.

\section{Acknowledgements}

The scientific research project of Hunan Provincial Education Department (Number: 15C0982).

\section{References}

[1] PENG Liang yu, HE Yi gang, HUANG Man chi1.The general two order voltage mode filter based on CFA [J].Journal of Naturnal Science of Hunan Normal University,2001,24(1):28-30.

[2] Jiun-Wei Horng , Maw-Huei Lee.High input impedance voltage-mode lowpass, bandpass and highpass filter using current-feedback amplifiers[J].Electronics Letters , 1997 , 33(11):947-948.

[3] Jiun-Wei Horng.New configuration for realizing universal voltage-mode filter using two current feedback amplifiers[J].IEEE Transactions on Instrumentation and Measurement , 2000 , 49(5):1043-1045.

[4] Shen-Iuan Liu, Dong-Shiuh Wu.New current-feedback amplifier-based universal biquadratic filter[J].IEEE Transactions on Instrumentation and Measurement , 1995 , 44(4):915-917.

[5] Chang C-M , Hwang C-S.Comment on "Voltage-mode notch, lowpass and bandpass filter using current-feedback amplifiers"[J].Electronics Letters , 1995 , 31(4):246.

[6] Liu S-I. Universal filter using two current-feedback amplifiers[J]. Electronics Letters , 1995 , 31(8):629-630. 